\title{
Die Verstetigung von urbanen Reallaboren im Spannungsfeld theoretischer Konzeption und praktischer Umsetzung - eine empirische Untersuchung
}

\author{
Nora Schecke, Abeer Abdulnabi Ali, Anna Bönisch, Stefan Schweiger \\ Eingegangen: 5. September 2020 - Angenommen: 6. April 2021 - Online veröffentlicht: 28. April 2021
}

\begin{abstract}
Zusammenfassung
Die Verstetigung urbaner Reallabore kann einen substanziellen Beitrag zu einer gelingenden sozialökologischen Transformation leisten. Die Umsetzung steht dabei Herausforderungen gegenüber, die unter anderem in der zeitlichen Fixierung der Projektförderung und der Komplexität transdisziplinärer Forschungslogik und -praxis liegen. Im Rahmen einer Untersuchung ausgewählter Drittmittelprojekte wurden Ausprägungen der Themenfelder Verstetigung, Transferierbarkeit, Beitrag zur Transformation, Reflexivität und Lernprozesse sowie Transdisziplinarität erhoben und eruiert, wie sich der Projektrahmen auf Verstetigung auswirkt. Die Befunde weisen auf eine tendenziell eher gelingende Fortsetzung der Kooperationen der unterschiedlichen Akteure hin; auch eine strukturelle
\end{abstract}

Nora Schecke, Geographisches Institut, Arbeitsgruppe Gesellschaft und Nachhaltigkeit im Wandel, Ruhr-Universität Bochum, Universitätsstraße 150, 44801 Bochum, Deutschland nora.schecke@rub.de

Abeer Abdulnabi Ali, Geographisches Institut, Arbeitsgruppe Gesellschaft und Nachhaltigkeit im Wandel, Ruhr-Universität Bochum, Universitätsstraße 150, 44801 Bochum, Deutschland abeer.abdulnabiali@rub.de

Anna Bönisch, Geographisches Institut, Arbeitsgruppe Gesellschaft und Nachhaltigkeit im Wandel, Ruhr-Universität Bochum, Universitätsstraße 150, 44801 Bochum, Deutschland anna.boenisch@rub.de

Stefan Schweiger, Geographisches Institut, Arbeitsgruppe Gesellschaft und Nachhaltigkeit im Wandel, Ruhr-Universität Bochum, Universitätsstraße 150, 44801 Bochum, Deutschland stefan.schweiger@rub.de

(c) (1) () 2021 Schecke; licensee oekom verlag. This Open Access article is published under a Creative Commons Attribution 4.0 International License.
Weiterführung der Reallaboraktivität durch Praxisakteure ist in verschiedenen Konstellationen möglich. Hinderlich ist das Ausbleiben der durch die Förderung gewährleisteten Infrastrukturen und Ressourcen im Zuge kurzer Projektlaufzeiten. Die Kriterien transdisziplinärer Forschung gehen mit zusätzlichen Anforderungen an die Wissenschaftler/-innen einher, welche wiederum den Erkenntniszuwachs in der Methodenentwicklung und -anwendung befördern, jedoch ebenfalls in Zusammenhang eines niedrigeren Outputs an Peer-ReviewAufsätzen zu bewerten sind.

Schlüsselwörter: Reallabor - Verstetigung sozialökologische Transformation - Transdisziplinarität Ökologie - Nachhaltigkeit

\section{The Continuation of Urban Real-World Laboratories between Theoretical Conception and Practical Implementation - An Empirical Study}

\begin{abstract}
The continuation and stabilization of urban real-world laboratories has the potential to contribute substantially to a successful socio-ecological transformation. Implementing actors face challenges which include time constraints related to project funding and the complexity of transdisciplinary research logic and practice. Selected externally funded research projects are assessed in terms of continuity, transferability, contribution to transformation, reflexivity and learning processes and transdisciplinarity to determine how project frameworks affect the continuity of real-world laboratory elements. The findings indicate a tendency towards a successful continuation of multi-stakeholder collaborations. A structural
\end{abstract}


continuation of real-world laboratory activities by practitioners is also proven to be possible in various constellations. The termination of donor funded infrastructure and resources remains a major obstacle due to short project durations. The criteria of transdisciplinary research are accompanied by additional demands on the scientists, which in turn promote the increase in knowledge of method development and application, but must also be considered in the context of a lower output of peer-reviewed articles.

Keywords: Real-world laboratories = Continuation - Socioecological transformation - Transdisciplinarity - Ecology * Sustainability

\section{Reallabore: Ein dynamisches Format transformativer Forschung}

Reallabore in wissenschaftlichen Drittmittelkontexten erschaffen in Intention, Problemidentifikation, Prozess und Funktion alternativ genutzte Räume, die - jenseits von Markt und Staat - ein Erproben von Zukunft (Diethard/ Höflehner 2018: 11) ermöglichen. Dies geschieht nicht nur, aber vor allem in den sich verdichtenden, funktional immer stärker vordefinierten, vom materiellen Metabolismus (Karvounis 2015: 3-12) und insbesondere von Arbeits- und Konsumvorgängen geformten (Harvey 2013: 93) und homogenisierten Städten, deren ,kulturelle Eigenarten“ (WBGU 2016: 153) entweder durch Stadtmarketing ökonomisiert werden oder sich aus anderen Gründen in unterschiedlichen Schwundstufen befinden (Augé 2011: 126-128). (Städtische) Probleme werden auf der Makroebene in Ausschreibungen von Ministerien oder Stiftungen weit definiert und in Reallaborprojekten auf der Mikroebene, z. B. Quartieren, identifiziert. Dort werden vor Ort prozessual partizipativ (Meyer-Soylu/Parodi/Trenks et al. 2016: 33-34), also auch transdisziplinär, unter Zuhilfenahme von Moderation und Expertise, kreative (Wagner 2017: 79-94) ortsangepasste Lösungspfade erarbeitet, ausgewählt und bottomup umgesetzt. Dieses ergebnisoffene Vorgehen (Schäpke/ Stelzer/Bergmann et al. 2017: 7) kann Räume schaffen, die ein lokal geteiltes Narrativ tradieren, vorausgesetzt die Projekte haben und verwirklichen die Möglichkeit zur Verstetigung.

Das Forschungsformat Reallabor hat eine relativ junge Historie (Beecroft/Trenks/Rhodius et al. 2018: 75; Grunwald/Schäfer/Bergmann 2020: 113) in der methodologi- schen Bestimmung und wissenschaftlichen Anwendung. ${ }^{1}$ Während sich bislang in der wissenschaftlichen Debatte keine Definition durchsetzen konnte, gibt es doch Überschneidungen sowie Übereinstimmungen in zentralen Merkmalen der in den letzten Jahren entwickelten Definitionen (Rose/ Wanner/Hilger 2019: 3). Um den Reallaborbegriff zu schärfen, haben Schäpke, Stelzer, Caniglia et al. (2018) aus dem gegenwärtigen Diskurs fünf allgemeine Charakteristika von Reallaboren abgeleitet, die von verschiedenen Wissenschaftlerinnen und Wissenschaftlern aufgegriffen wurden (z. B. Rose/Wanner/Hilger 2019; Wanner/Stelzer 2019) und an denen sich die Autorinnen und Autoren im vorliegenden Beitrag ebenfalls orientieren:

- Beitrag zur Nachhaltigkeitstransformation

- (Real)experimente als methodische Grundlage

- Transdisziplinärer Forschungsmodus

- Langzeitorientierung, Skalierbarkeit und Transferierbarkeit $^{2}$

- Reflexions- und Lernprozesse

Bezüglich der potenziellen Orte für Reallaborprojekte gibt es kaum Einschränkungen (Schäpke/Stelzer/Bergmann et al. 2017: 4), gleichwohl ist ein klar definierter Bezugsraum wichtig, der von den beteiligten Akteuren anerkannt wird und der mit der inhaltlichen Ausrichtung vereinbar ist (Beecroft/Trenks/Rhodius et al. 2018: 86-87). Abgrenzungen können unter anderem nach funktionalen (z. B. Pendlerverkehr einer Stadt), administrativen (Stadt, Stadtquartier) oder naturräumlichen Kriterien (Naturraum wie etwa der Nordschwarzwald) erfolgen (Beecroft/Trenks/ Rhodius et al. 2018: 87). Deutlich wird, dass Reallabore komplexe dynamische Eigenschaften auf methodischer sowie theoretischer Ebene vereinen (Defila/Di Giulio 2018: 9): Für realweltliche Probleme sollen Lösungen bzw. Innovationen im Kontext von Realexperimenten (,Experimental Turn“; Kügler/Ronnenberg 2017: 22) entwickelt und erprobt werden, welche sich durch eine transdisziplinäre und transformative Herangehensweise und Methodik kennzeichnen lassen (Schneidewind 2014: 2; Beecroft/ Trenks/Rhodius et al. 2018: 76). In Kooperation von Wis-

\footnotetext{
${ }^{1}$ Die (Güte-)Kriterien transformativer Forschung werden breit diskutiert: Schneidewind/Singer-Brodowski (2014), Strohschneider (2014), Grunwald (2015), Schneidewind (2015) und Wissenschaftsrat (2015).

2 Laut Rose, Wanner und Hilger (2019:4) wird die Transferierbarkeit als definitorisches Element noch „kontrovers diskutiert“.
} 
senschaftlerinnen/Wissenschaftlern ${ }^{3}$ und Praxisakteuren werden Forschungsziele mit Praxiszielen verknüpft, um System-, Ziel- und Transformationswissen für die Gestaltung (soziotechnischer) ${ }^{4}$ Transformationsprozesse zur Lösung lokal auftretender Probleme zu erzeugen sowie Wandlungsprozesse anzustoßen (WBGU 2011: 89-91, 99101, 342-343; Eckart/Ley/Häußler et al. 2018: 105). Dies erfolgt unter Anwendung eines strukturierten und reflexiv angelegten Ko-Designs und einer Ko-Produktion von Wissen (Schäpke/Stelzer/Bergmann et al. 2017: 19). Inhaltlich beziehen sich die Problemstellungen transformativer Forschung im Allgemeinen, wie auch von Reallaboren im Speziellen meist auf Aspekte nachhaltiger Entwicklung, es sind jedoch auch andere Fragestellungen mit dem Format Reallabor vereinbar, sofern sie gesellschaftlich legitimiert, ethisch gut begründet sowie gemeinwohlorientiert sind (Beecroft/Trenks/Rhodius et al. 2018: 77, 78; Defila/Di Giulio 2018: 11, 12). Ein Beispiel ist die Problemstellung der ,Steigerung von Teilhabemöglichkeiten gesellschaftlich benachteiligter Gruppen (z. B. in Ankunftsquartieren)“ (Räuchle/Schmiz 2019: 17). Die fokussierte Darstellung ausgewählter Eigenschaften von Reallaboren deutet es an: Das Forschungsformat Reallabor ist in seiner normativen Konstitution (Freier/Lübke/Schrot et al. 2020: 241242) und Idealvorstellung (Schneidewind/Singer-Brodowski 2015: 14-20) komplex (abzubilden beispielsweise in der Komplexität von Transformationsprozessen), heterogen (zu illustrieren anhand unterschiedlicher Problemstellungen, Anwendungskontexte, gewählter Methoden und teilnehmender wie auch betroffener Akteure) und dynamisch bzw. reflexiv (sichtbar durch den transdisziplinär ausgerichteten und fortwährend Lern- und Neujustierungsprozessen unterzogenen Forschungsprozess).

Aufgrund der oben beschriebenen Spezifika wird Reallaboren, unter anderem von der deutschen Forschungs- und Innovationspolitik (Deutscher Bundestag 2018), ein hohes Innovationspotenzial zugeschrieben, welches auf einen gewissen Trendcharakter projektbezogener Umsetzungen hinweist. Deutlich wird dies anhand von Förderlinien und Forschungsschwerpunkten auf nationaler wie internationaler Ebene. ${ }^{5}$ Dies legt die Frage nahe, wie sich die Projektstruk- tur im Drittmittelkontext auf die Umsetzung des jeweiligen Reallabors auswirkt. Welche Effekte hat der Projektrahmen auf Faktoren der Verstetigung von Reallaboren im Sinne eines Fortbestehens über die Projektlaufzeit hinaus? Wie lassen sich Offenheit und Reflexivität bezüglich der transdisziplinär zu bestimmenden Forschungsdesignentscheidungen in einem fixen Projektrahmen umsetzen? In der Projektrealität können mögliche Fallstricke des konzeptionellen Anspruchs in Bezug auf die Umsetzung von Projektbausteinen, mit all ihren monetären wie zeitlichen Fixierungen, sichtbar werden.

In diesem Beitrag werden die oben genannten Problemstellungen anhand ausgewählter Reallaborprojekte hinsichtlich folgender Fragen untersucht: Wie wirkt sich der drittmittelfinanzierte Projektrahmen auf die Verstetigung von Reallaboren aus? Welche förderlichen und hemmenden Bedingungen sind aus den Daten ableitbar? In Kapitel 2 werden der Untersuchungsgegenstand (Drittmittelprojekte) und das gewählte Mixed-Method-Design (standardisierte Befragung und Analyse von Dokumenten) genauer erläutert. Die Ergebnisse werden in Kapitel 3 präsentiert und im Fazit und Ausblick (Kapitel 4) im Hinblick auf die projektbezogene Umsetzbarkeit von Reallaboren diskutiert.

\section{Gegenstand und Methodik}

Die Methodik folgt einem explorativen Vorgehen. Um Vergleichbarkeit gewährleisten zu können, wurden Projektförderungen des Bundesministeriums für Bildung und Forschung $(\mathrm{BMBF})^{6}$ und des Landes Baden-Württemberg, unter anderem innerhalb der Förderlinien „Reallabore - BaWü-Labs“ und „Reallabore Stadt“ des Ministeriums für Wissenschaft, Forschung und Kunst (MWK), ausgewählt. ${ }^{7}$ Insgesamt umfasst die Stichprobe 28 Projekte mit unterschiedlichen Laufzeiten zwischen 2013 und 2020, ungeachtet etwaiger Verlängerungen. Aus diesem Sample wurden die Projekte zur genaueren Analyse ausgewählt, deren Abschlussberichte bzw. Ergebnisdokumentationen online zugänglich waren oder die diese auf Nachfrage zur Verfügung

\footnotetext{
${ }^{6}$ Vertreten waren unter anderem Projekte aus dem Förderschwerpunkt „Sozialökologische Forschung“.

7 Einen anschaulichen Einblick in ausgewählte bundesweit umgesetzte Reallaborprojekte bietet das Netzwerk Reallabore: https:// www.reallabor-netzwerk.de/mitgliederverzeichnis/reallabore-

projekte/ (12.03.2021).
}

\footnotetext{
3 Verstanden als Angehörige des Wissenschaftssystems. Es wird hier auf die Schwierigkeiten hingewiesen, welche mit der Begriffsbestimmung der Akteure im transdisziplinären Kontext einhergehen. Diese umfassen auch normative Implikationen (vgl. auch Erläuterungen bei Eckart/Ley/Häußler et al. 2018: 106-107).

${ }^{4}$ Vgl. hierzu vertiefend einschlägige Arbeiten zu soziotechnischen Wandlungsprozessen der Transition-Forschung, z. B. bei Geels/ Schot (2007), Grin/Rotmans/Schot (2010) und Geels (2011).

${ }^{5}$ Eine übersichtliche Darstellung ausgewählter deutscher Förderlinien findet sich bei Wanner/Stelzer (2019).
} 
gestellt haben. ${ }^{8}$ Für sieben Projekte lagen uns Berichte vor. ${ }^{9}$ Zusätzlich wurde Wissenschaftlerinnen und Wissenschaftlern aus den 28 Projekten ein Fragebogen zugesandt. Der Rücklauf ${ }^{10}$ betrug 12 beantwortete Bögen, vier davon aus Projekten, zu denen uns die Ergebnisdokumentationen vorlagen. Die Projekte, zu denen die Fragebögen eingeholt werden konnten, wurden der Auswahl hinzugefügt. Der Datenkorpus umfasst schlussendlich 15 Projekte ${ }^{11}$, fünf gefördert durch das BMBF, neun durch das MWK und eines durch das Ministerium für Umwelt, Klima und Energiewirtschaft des Landes Baden-Württemberg. ${ }^{12}$

Hinsichtlich des Inhalts, der disziplinären Ausrichtung und des räumlichen Zuschnitts sind die untersuchten Projekte vielfältig aufgestellt: In den meisten Fällen (4) wurde das Reallabor im Quartier umgesetzt, daneben wurden Städte (2), Stadtteile und -bezirke (2) und jeweils einmal kleinräumige Wohnsiedlungen, Unternehmen und andere private wie auch öffentliche Einrichtungen genannt. Inhaltlich werden Themen wie Mobilität, ganzheitliche Stadtentwicklung und Nachhaltigkeit, Bildung sowie Produktion, Vertrieb und Konsum bearbeitet (disziplinär schwerpunktmäßig von Wissenschaftlerinnen/Wissenschaftlern aus den Forschungsrichtungen der Sozial- und Geisteswissenschaf-

\footnotetext{
8 Dennoch sollte Form und Inhalt derartiger Berichte nicht unreflektiert bleiben. Zum einen variieren diese in Länge und Ausführlichkeit. Zum anderen können unterschiedliche Gewichtungen in der Reflexion und Darstellung von Problematiken und Schwierigkeiten während der Projektlaufzeit vermutet werden. Hierzu fehlt das entsprechende Kontextwissen, was im Rahmen einer Dokumentenanalyse zu beachten ist (Döring/Bortz 2016: 537).

9 Der Bitte zum Erhalt des Abschlussberichtes konnte zum Teil aufgrund von formellen wie auch informellen Hürden nicht nachgekommen werden. Solche Hürden äußerten sich in datenschutzrechtlichen Festlegungen oder Unsicherheiten, einer noch nicht abgeschlossenen Schlussdokumentation oder beispielsweise im Aufwand der Absprache mit dem Fördergeber oder den Verbundpartnern.

10 Die Analyseergebnisse sind insofern nur eingeschränkt aussagekräftig, als dass nicht ausgeschlossen werden kann, dass vor allem Projekte, die hinsichtlich unterschiedlicher Ausprägungen von Verstetigung nicht erfolgreich waren, nicht auf die Bitte um $\mathrm{Zu}$ sendung des Projektberichts sowie um Beantwortung des Fragebogens reagierten.

11 Fünf der untersuchten Projekte wurden im Jahr 2020 abgeschlossen (2019: vier Projekte, 2018: fünf Projekte, 2017: ein Projekt). Im Fall von Projekten mit zwei Förderphasen wurde der Abschluss der letzten Phase genannt. Anzumerken ist hier, dass bei einem erst kürzlich abgeschlossenen Projekt die untersuchten Verstetigungsausprägungen schwieriger zu bewerten sind.

12 Bezüglich datenschutzrechtlicher Festlegungen wurde den einzelnen Projekten zugesichert, in der Ergebnisaufbereitung keine Benennung einzelner Projekte, institutioneller Zugehörigkeiten und Personen und somit keine exemplarischen Fallbeschreibungen vorzunehmen.
}

ten sowie den Ingenieur-, Wirtschafts- und Naturwissenschaften).

Im Fragebogen wurden Ausprägungen der Themenfelder Verstetigung, Transferierbarkeit, Beitrag zur Transformation, Reflexivität und Lernprozesse sowie Transdisziplinarität erhoben. Der Fokus lag auf den Ausprägungen von Verstetigung des jeweiligen Reallabors. Die weiteren Themenfelder dienten der Analyse, um Wechselwirkungen zwischen diesen und der Verstetigungskategorie eruieren zu können. Die Themenfelder wurden auf der Grundlage des aktuellen Forschungsstands zu Reallaboren und dem Wissenschaftsverständnis transdisziplinärer Forschung entwickelt (Jahn 2013; Parodi/Beecroft/Albiez et al. 2016: 11, 16-17; Schäpke/Stelzer/Bergmann et al. 2017) und in den Fragebogen mittels standardisierter wie offener Fragen überführt. Die Spezifikation der inhaltlich zu erfassenden Ausprägungen von Verstetigung bildete den Schwerpunkt der theoretischen Vorarbeit. Hierzu wurden heuristische Kategorien gebildet, welche Verstetigung in strukturelle, kooperative, praktische und wissenschaftliche Verstetigung differenzierten. Die Einteilung dient einer kategoriengeleiteten Ausdifferenzierung inhaltlicher Facetten von Verstetigung, wobei allerdings kein Anspruch auf Vollständigkeit besteht. Wir hoffen, damit weitere Forschungstätigkeit anzuregen.

Die strukturelle Verstetigungskategorie umfasst Elemente der Fortführung der Reallabore im Sinne einer Etablierung von finanziellen Trägerstrukturen, einer durch Drittmittel finanzierten Anschlussförderung sowie örtlich fortbestehender und nutzbarer Räumlichkeiten. Möglich sind unterschiedliche Trägerstrukturen: „Reallabore sind diesbezüglich nicht festgelegt und könnten sowohl durch Institutionalisierung an bspw. Hochschulen oder durch Übergang in gesellschaftliche Trägerschaft verstetigt werden“" (Schäpke/Stelzer/Bergmann et al. 2017: 47). Eine kritische Befassung mit Divergenzen zwischen einer Prozessinitiierung, der transdisziplinären Umsetzbarkeit im Förderzeitraum sowie einer langfristigen Verstetigung über diesen hinaus findet sich bereits im wissenschaftlichen Diskurs über Reallabore (Schäpke/Stelzer/Bergmann et al. 2017: 7, 47; Rose/Wanner/Hilger et al. 2019: 22). Die kooperative Verstetigungskategorie umfasst die Zusammenarbeit zwischen Wissenschaftlerinnen/Wissenschaftlern und/oder Praxisakteuren, worunter insbesondere Akteure aus Zivilgesellschaft, Politik, Verwaltung und Wirtschaft fallen, sowie neu entstandene Netzwerke und Formen der Zusammenarbeit. Derartige Kooperationen bilden ein Kernkriterium von Inter- und Transdisziplinarität (Schäpke/Stelzer/ Bergmann et al. 2017: 21-22, 47; Puttrowait/Dietz/Gantert et al. 2018: 195-231; Best 2020: 97). Unter einer praktischen Verstetigung wird die Überführung der Lösungen und Ergebnisse in die Lebenswelt verstanden. Relevant ist hier die Anwendung von Innovationen und/oder Produkten 
durch Praxisakteure sowie deren gelungene Adressierung seitens des Projektteams (Schäpke/Stelzer/Bergmann et al. 2017: 40, 48). Schließlich ist in Bezug auf wissenschaftliche Verstetigungsausprägungen die Verwertung von und Auseinandersetzung mit den Reallaborergebnissen im wissenschaftlichen Bereich relevant, was die Diskussion und damit Verbesserung und Verfeinerung durch die scientfic community vorantreibt. Dazu zählt insbesondere, Reallabore auf theoretisch-konzeptioneller wie auch evaluativer Ebene weiterzuentwickeln (Beecroft/Parodi 2016: 7).

Die Abschlussberichte bzw. Ergebnisdokumentationen wurden anhand dieser Kategorien unter Anwendung einer strukturierenden qualitativen Inhaltsanalyse untersucht (Mayring 2010). Es wurde ein Mixed-Methods-Forschungsdesign realisiert, welches quantitative (Fragebogenentwicklung) und qualitative (Untersuchung der Berichte, Dokumentationen) Erhebungs- und Analysemethoden verbindet (Schreier/Odağ 2010: 263-277).

\section{Wie steht es um die Verstetigung?}

Im Folgenden werden die Ergebnisse der standardisierten Befragung vorgestellt. Sofern in den Abschlussberichten der drei Reallabore, die nicht an der Befragung teilgenommen haben, Informationen zu den verschiedenen Fragen vorlagen, werden diese der Ergebnisdarstellung hinzugefügt. ${ }^{13}$

\subsection{Strukturelle Verstetigung}

Die Ergebnisse zur strukturellen Verstetigung zeichnen ein heterogenes Bild (vgl. Tabelle 1).

Laut der Befragten und der Informationen aus den Berichten bestehen acht von 13 Reallaboren nach Projektende fort. Dies wurde jedoch in geringerer Ausprägung (6 von 11) innerhalb der im Durchführungszeitraum finanzierten Räumlichkeiten umgesetzt. Die Variation der Fallzahlen geht auf Non-Response im Fragebogen und Ergänzungen aus der Analyse der Berichte zurück. Die Fortführung wurde in der Mehrzahl nicht durch eine Anschlussförderung (9 von 12) oder durch eine etablierte Trägerstruktur außerhalb der Drittmittelfinanzierung (7 von 11) realisiert. In zehn von 12 Fällen wurde dies durch eine eigenverantwortliche Steuerung der Praxisakteure übernommen. Die Daten zeigen, dass die informelle Fortführung von (einzelnen) Aktionen vor allem an das Engagement der (Praxis-)Akteure gekoppelt ist. Wie sich die eigenverantwortliche Steuerung konkret umsetzt, kann ohne detaillierte Ausführungen der

\footnotetext{
13 Dies wird unter den Tabellen kenntlich gemacht.
}

Tabelle 1 Ausprägungen einer strukturellen und kooperativen Verstetigung (Daten aus Fragebögen und Berichten)

\begin{tabular}{|c|c|c|}
\hline $\begin{array}{l}\text { Fragen zur strukturellen und kooperativen Ver- } \\
\text { stetigung des Reallabors }\end{array}$ & Ja & Nein \\
\hline Besteht das Reallabor nach Projektende fort? & 8 & 5 \\
\hline Können Räumlichkeiten weitergenutzt werden? & 6 & 5 \\
\hline $\begin{array}{l}\text { Wurde das Reallabor durch eine Anschlussför- } \\
\text { derung verlängert? }\end{array}$ & 3 & 9 \\
\hline $\begin{array}{l}\text { Wurden finanzielle Trägerstrukturen außerhalb } \\
\text { der Drittmittelförderung etabliert? }\end{array}$ & 4 & 7 \\
\hline $\begin{array}{l}\text { Wurden Personen aktiviert, die sich über die } \\
\text { geförderte Projektlaufzeit hinaus weiter für die } \\
\text { Themen engagieren? }\end{array}$ & 14 & 1 \\
\hline $\begin{array}{l}\text { Wurden Kooperationen/Netzwerke im Realla- } \\
\text { bor gebildet, welche nach Projektende weiter } \\
\text { bestehen? }\end{array}$ & 14 & 0 \\
\hline $\begin{array}{l}\text { Wurde es in Eigenverantwortung durch die be- } \\
\text { teiligten Praxisakteure fortgeführt? }\end{array}$ & 10 & 2 \\
\hline
\end{tabular}

Befragten nicht abgeleitet werden. Die Antworten auf die offen gestellten Fragen bilden folgende Konkretisierung ab: Die verantwortlichen Akteure sind überwiegend den Sektoren Zivilgesellschaft und Verwaltung zuzuordnen. Eine formelle Verankerung in Form einer finanziell verpflichtenden Trägerschaft oder Anschlussförderung ist dagegen seltener. Sofern jedoch eine formelle Verankerung bestand, wurde die Trägerschaft unter anderem in Form von städtischen Zuschüssen oder Verankerungen in Einrichtungen der Stadtverwaltungen, einer Beteiligung durch Eigenanteile der Projektpartner/-innen, wirtschaftlicher Geschäftsmodelle oder mittels der Kopplung an eine Hochschule oder eine andere wissenschaftliche Einrichtung umgesetzt. Eine Fortführung mit dem Schwerpunkt auf örtlich frequentierte Räumlichkeiten war nicht immer bezweckt, da die Räumlichkeiten nur für die begrenzte Zeitspanne des Experimentierens vorgesehen waren oder zur Durchführung keine Räume benötigt wurden.

Die Ergebnisse schließen an die Diskussion über die Umsetzung von Reallaboren in Projektkontexten an wie bei Schäpke, Stelzer, Bergmann et al. (2017: 7): ,Zugleich wird der begrenzte Zeithorizont der Projekte hinsichtlich geplanter Interventionen oder für den Vertrauens- und Strukturaufbau als Herausforderung betrachtet. Ebenso ist die Finanzierung des weiteren Verlaufs der Reallabore und ihre Verstetigung ungeklärt. Dieses betrifft besonders die (eventuelle) Ungleichzeitigkeit zwischen dem formalen Projektende und den angestoßenen bzw. (weiter-) laufenden Prozessen.“ Die Daten, die zur Identifikation von Gründen für eine NichtVerstetigung (7 von 12 Ausführungen im offenen Fragenblock) dienen, bilden die zeitlichen und strukturellen $\mathrm{Be}$ sonderheiten des Reallaborformats ab: Nach Ansicht der Befragten wäre eine längere Projektdauer wünschenswert 
gewesen, da sich die knapp bemessene Länge der Projekte hinderlich auf eine strukturelle Etablierung der Reallaborlösungen und -tätigkeiten ausgewirkt hat, was auch den Erfordernissen transdisziplinärer Projektarbeit in Form eines zeitintensiven Aufbaus von vertrauensvollen Beziehungen sowie der zeitaufwendigen Arbeit in der Praxis geschuldet ist.

\subsection{Kooperative Verstetigung}

Im Hinblick auf eine kooperative Verstetigung zeichnet sich nach Auswertung der Fragebögen und der drei Berichte ein durchweg positives Meinungsbild ab. In nahezu allen Fällen wurden Personen aktiviert, die sich über die geförderte Projektlaufzeit hinaus weiter für die Themen engagieren (14 von 15). Gänzlich zugestimmt wurde der Aussage, dass Kooperationen eingegangen/Netzwerke gebildet wurden, von denen angenommen werden kann, dass sie auch nach Projektende Bestand haben könnten (14 von 14). Nach den Angaben der Befragten entstanden übergreifende Kooperationen mehrheitlich mit Akteuren aus der Zivilgesellschaft, gefolgt von jenen aus der Verwaltung, der Wissenschaft und schlussendlich der Wirtschaft (insgesamt lagen zehn Antworten im offenen Fragenblock vor). Eine Aktivierung der (Praxis-)Akteure wurde nach Meinung der Befragten erreicht, was als Voraussetzung eines anzustoßenden sozialökologischen Transformationsprozesses gilt (WBGU 2011: 256-260). Nach Beecroft, Trenks, Rhodius et al. (2018: 80) werden langfristige Kooperationsbeziehungen sowie ein gelungenes Empowerment der Praxisakteure einzelnen Praxiszielen zugeordnet („Kooperationsziel“ und „Empowermentziel“). Das Kooperationsziel - hier als kooperative Verstetigungsausprägung erfasst - kann laut Einschätzung der Befragten und unter Rückgriff auf die Berichte als erfüllt bewertet werden.

Kritisch zu bedenken sei die Beurteilung von Kooperationen im zeitlichen Verlauf: Es kann nichts darüber ausgesagt werden, inwieweit die befragten Wissenschaftler/-innen aktuelle Informationen zu Art, Umfang und Aufrechterhaltung der Kooperationen vorliegen haben. Ob mit der stattgefundenen Aktivierung von Personen auch ein Empowerment ${ }^{14}$ einhergegangen ist, kann aus den Ergebnissen ebenfalls nicht geschlossen, jedoch als förderliches Verstetigungsmerkmal herausgestellt werden: „Empowerment wird in einem Re-

\footnotetext{
${ }^{14}$ Empowerment wird auf der letzten Intensitätsstufe eines partizipativen Prozesses (nach Information, Konsultation, Kooperation, Kollaboration) angesiedelt (Meyer-Soylu/Parodi/Trenks et al. 2016: 33, 36-38; Beecroft/Trenks/Rhodius et al. 2018: 80-82; in Anlehnung an Arnstein 1969 und Brinkmann/Bergmann/ Huang-Lachmann et al. 2015).
}

allabor angestrebt, damit die Akteure - sowohl aus der Praxis wie aus der Wissenschaft - in die Lage versetzt werden, auch über die Laufzeit der transdisziplinären Projekte hinaus an den Transformationsprozessen langfristig aktiv teilhaben zu können“ (Beecroft/Trenks/Rhodius et al. 2018: 82).

\subsection{Praktische Verstetigung}

Die im Rahmen der Reallabore entwickelten Lösungen und Methoden wurden laut der Befragten in hohem Maße öffentlichkeitswirksam ( 6 von $12=$ stimme zu, 3 von 12 = stimme eher zu) verbreitet. Lediglich bei drei von 12 wurde eher keine Zustimmung angegeben. In Berichten zwei weiterer Reallabore, für die keine ausgefüllten Fragebögen vorliegen, wird eine öffentlichkeitswirksame Berichterstattung ebenfalls betont. Die Projektergebnisse werden größtenteils durch Pressearbeit und Online-Medien (Social Media, Homepage), Berichte, Workshops und Vorträge in die Öffentlichkeit getragen. Nach Projektende können unterschiedliche Produkte bzw. Lösungen (darunter Plattformen, Konzepte für nachhaltigen Konsum, Informationsmaterialien/Factsheets und Schulungsmaterialien, Agendaprozesse, Handlungsleitfäden) potenziell von den Praxisakteuren weiter genutzt werden. Dies erschließt sich aus sechs Antworten aus den Fragebögen und kann nach dem Stand der Berichte um fünf weitere Reallabore ergänzt werden. Inwieweit die erarbeiteten Lösungen bzw. Methoden tatsächlich Anwendung fanden, kann im Rahmen dieser Studie nicht geschlossen werden. Die thematische Ausrichtung gestaltet sich vielseitig. Sie erstreckt sich von Förderung erneuerbarer Energien über Stadt-/Quartiersentwicklung bis hin zu nachhaltiger Textilwirtschaft.

Eine öffentlichkeitswirksame Berichterstattung über die entwickelten Lösungen kann den narrativen Charakter von Realexperimenten (Puttrowait/Dietz/Gantert et al. 2018: 197) fördern, wodurch Möglichkeiten entstehen, die Transferierbarkeit zu verbessern. Insofern kann die mediale Berichterstattung förderlich für die praktische Verstetigung von Reallaboren sein. Dies hängt allerdings stark von der narrativen Ausgestaltung ab: Diese sollte sich nicht in Formen des Marketings im Sinne einer Produktion warenförmiger Bedürfnisse verlaufen, um nicht den Anschein von Persuasion zu erzeugen, sondern transparent die Beteiligungsmöglichkeiten narrativisieren, mit dem Ziel, für das jeweilige Projekt Begeisterung zu wecken. $\mathrm{Zu}$ bedenken sind außerdem Anforderungen an eine angemessene Sprache auf allen Ebenen der Zusammenarbeit und Adressierung verschiedener Ziel- und Akteurgruppen (Eckart/Ley/Häußler et al. 2018: 109). Beecroft und Parodi (2016) erläutern, dass die praktische Ausgestaltung der Realexperimente sowie der entwickelten Lösungen durch 
Tabelle 2 Beitrag der Reallabore zum wissenschaftlichen Output (Daten aus Fragebögen)

\begin{tabular}{lllll}
\hline \multicolumn{6}{l}{$\begin{array}{l}\text { Wie würden Sie den wissenschaftlichen Output, der aus dem } \\
\text { Projekt/Reallabor generiert wurde, quantifizieren? }\end{array}$} \\
\hline & $\begin{array}{l}\text { sehr } \\
\text { hoch }\end{array}$ & hoch & gering & $\begin{array}{l}\text { kein } \\
\text { Output }\end{array}$ \\
\hline $\begin{array}{l}\text { Aufsätze (Peer-Review) } \\
\text { Aufsätze (ohne Peer- }\end{array}$ & 0 & 5 & 7 & 0 \\
Review) & 1 & 7 & 4 & 0 \\
Working Paper & 2 & 3 & 4 & 1 \\
Vorträge & 6 & 6 & 0 & 0 \\
Sonstiges & 3 & 0 & 0 & 0 \\
\hline
\end{tabular}

die Schaffung einer Anlaufstelle gefördert werden kann, damit Interessierte das Reallabor ,aktiv aufsuchen, weitere Experimente oder Aktionen anstoßen oder letzten Endes an der Gestaltung des Reallabors selbst mitwirken“ (Beecroft/ Parodi 2016: 7) können.

\subsection{Wissenschaftliche Verstetigung}

Die Fragen nach der Ergebnisverwertung, dem wissenschaftlichen Output sowie dem theoretischen und methodischen Beitrag zur Forschung zeichnen ein durchmischtes Bild. Auffällig ist, dass die Ergebnisse in 13 von 14 Fällen in weitere (Folge-)Anträge geflossen sind. Darin kann eine Chance für Wissenschaftler/-innen in der transformativen Forschung verortet werden, dem vorliegenden Forschungsdesiderat auch zukünftig nachgehen und das persönliche Fortkommen sowie das der scientific community fördern zu können.

Dem Datenmaterial zufolge konnte wissenschaftlicher Output in allen Projekten in Veröffentlichungen, darunter Aufsätze (ohne und mit Peer-Review), Working Paper, Methodenhandbücher, Politik-Empfehlungen und Vorträgen, generiert werden (vgl. Tabelle 2). Die Quantifizierung des Outputs fiel jedoch unterschiedlich aus. Hieraus ergibt sich eine Problematik, die im Fazit und Ausblick diskutiert wird.

Peer-Review-Aufsätze wurden in sieben von 12 Fällen mit geringem Output und in fünf von 12 Fällen mit hohem Output eingestuft. Dahingegen wurden Aufsätze ohne PeerReview in sieben von 12 Fällen mit hohem Output sowie mit sehr hohem in einem von 12 Fällen und geringem Output in vier von 12 Fällen bewertet. Working Paper wurden mit sehr hohem (2 von 10), hohem (3 von 10), geringem (4 von 10) und keinem Output (1 von 10) quantifiziert. Vorträge hingegen haben sechs von 12 jeweils mit sehr hohem und hohem Output eingestuft. Darüber hinaus wurden Buchpublikationen zu Projektmethoden und Nachhaltigkeit sowie politische Handlungsempfehlungen als Output unter Sonstiges von drei Befragten genannt. Eine höhere Zustim- mung ( 7 von $12=$ stimme $\mathrm{zu}, 5$ von $12=$ stimme eher zu) wurde für den Beitrag der Projektergebnisse zur methodischen Entwicklung von Reallaboren im Vergleich zum Beitrag der Projektergebnisse zum wissenschaftlich-theoretischen Diskurs transdisziplinärer Forschung (5 von $12=$ stimme $\mathrm{zu}$ und stimme eher $\mathrm{zu}, 2$ von $12=$ stimme eher nicht zu) geäußert. Inhaltlich konnten die Ausprägungen des methodischen und theoretischen Beitrags in einem offenen Fragenblock spezifiziert werden. Angegeben wurde von vier Befragten unter anderem ein Erkenntniszuwachs in der transdisziplinären Forschungspraxis, insbesondere bezogen auf Methoden sowie die Ausgestaltung der Zusammenarbeit und Rolle von Wissenschaft und Praxis bzw. verschiedener Akteursgruppen und Stakeholder. Dieser Mehrwert lässt sich schwerlich durch einen quantifizierbaren wissenschaftlichen Output abbilden und veranschaulicht die vielschichtigen Anforderungen transdisziplinärer Forschung.

Es gilt zu untersuchen, ob die transdisziplinäre Forschungsrichtung entweder Ergebnisse generiert, die sich nicht für begutachtete Aufsätze eignen, die Auslastung der Wissenschaftler/-innen zu hoch ist (Rose/Wanner/Hilger et al. 2019: 26) oder die Ergebnisdarstellung größtenteils nichtwissenschaftliche Kooperationspartner/-innen adressiert. Es ergeben sich unter Umständen Nachteile für Nachwuchswissenschaftler/-innen. Unseres Erachtens wäre es geboten, nicht die Forschungsmethode den Kriterien des Review-Prozesses anzupassen, sondern wissenschaftlich anerkannte Prozedere mit Bewertungskriterien für lebensnahe Forschung zu entwickeln. Die Lösung sollte nicht sein, einen Störfaktor Mensch gelten zu lassen, der die Ergebnisse allzu sehr verfälscht. Die Gefahr bestünde sonst darin, nicht nur Wissenschaftler/-innen eines bestimmten Forschungszweigs in der Diskussion der scientific community außen vor zu lassen, sondern einen Forschungsgegenstand zu übersehen. Unser Vorschlag wäre, den Forschungskonsortien weitgehend unabhängige Wissenschaftler/-innen, z. B. aus der Ethnologie, zur Seite zu stellen, die das Projekt deskriptiv und konsultativ begleiten, so einen intersubjektiven Evaluationsprozess zu ermöglichen und die Methodik sowie den tatsächlichen Verlauf plausibler und glaubwürdiger in den wissenschaftlichen Diskurs einzuspeisen.

\subsection{Transferierbarkeit}

Die Übertragbarkeit der Lösungen auf andere Untersuchungs- und Problemkontexte (Transferierbarkeit) erhält breite Zustimmung ( 3 von $12=$ stimme zu, 7 von $12=$ stimme eher zu) (vgl. Tabelle 3). Gleichwohl ist Transferierbarkeit, aufgrund des kontextspezifischen Vorgehens von Reallaboren, abhängig von den jeweiligen Realexperimenten bzw. Problemlösungen sowie den zugrunde liegenden 
Tabelle 3 Aussagen zu Transferierbarkeit der in den Reallaboren entwickelten Lösungen (Daten aus Fragebögen)

\begin{tabular}{|c|c|c|c|c|}
\hline Transferierbarkeit & & & & \\
\hline & stimme zu & $\begin{array}{l}\text { stimme eher } \\
\text { zu }\end{array}$ & $\begin{array}{l}\text { stimme eher } \\
\text { nicht zu }\end{array}$ & $\begin{array}{l}\text { stimme } \\
\text { nicht zu }\end{array}$ \\
\hline $\begin{array}{l}\text { Eine Übertragung der entwickelten Lösungen auf andere Untersu- } \\
\text { chungskontexte ist möglich. }\end{array}$ & 3 & 7 & 1 & 1 \\
\hline Es wurden primär fallspezifische Lösungen entwickelt. & 4 & 3 & 4 & 0 \\
\hline $\begin{array}{l}\text { Es ist nachvollziehbar, wie die Ergebnisse in nichtwissenschaftliche } \\
\text { Praxisfelder überführt werden können. }\end{array}$ & 3 & 8 & 0 & 0 \\
\hline
\end{tabular}

Tabelle 4 Beitrag zur Transformation (Daten aus Fragebögen)

\begin{tabular}{|c|c|c|c|c|}
\hline \multicolumn{5}{|l|}{ Beitrag zur Transformation } \\
\hline & stimme zu & $\begin{array}{l}\text { stimme eher } \\
\text { zu }\end{array}$ & $\begin{array}{l}\text { stimme eher } \\
\text { nicht zu }\end{array}$ & $\begin{array}{l}\text { stimme } \\
\text { nicht zu }\end{array}$ \\
\hline Durch das Reallabor wurden Transformationsprozesse angestoßen. & 3 & 7 & 1 & 0 \\
\hline $\begin{array}{l}\text { Das Reallabor wurde zum Zweck einer konkreten Problemlösung (als } \\
\text { Beitrag zur Transformation) durchgeführt. }\end{array}$ & 3 & 4 & 3 & 1 \\
\hline $\begin{array}{l}\text { Über das Anstoßen von langfristigen Transformationsprozessen kön- } \\
\text { nen keine validen Aussagen getroffen werden. }\end{array}$ & 1 & 6 & 3 & 0 \\
\hline $\begin{array}{l}\text { Die Forschungsziele konnten nicht gänzlich mit den Praxiszielen aus- } \\
\text { balanciert werden. }\end{array}$ & 0 & 5 & 4 & 1 \\
\hline
\end{tabular}

Fragestellungen. Über die Aussagekraft der Transferierbarkeit kann an dieser Stelle folglich nur begrenzt Auskunft gegeben werden; ob und wie der Transfer empirisch erprobt oder umgesetzt wurde, kann nicht beurteilt werden.

Primär fallspezifische Lösungen wurden - nach Aussagen der Befragten - hingegen von der Mehrheit der Reallabore entwickelt $(4$ von $11=$ stimme zu, 3 von $11=$ stimme eher $\mathrm{zu}, 4$ von $11=$ stimme eher nicht zu). In elf Fällen kann nachvollzogen werden, wie die Ergebnisse in nichtwissenschaftlichen Praxisfeldern angewendet werden können. Das aktuell noch junge Format Reallabor und seine Heterogenität erfordern eine Evaluationspraxis, die es ermöglicht, die Übertragbarkeit der Lösungen zu untersuchen und entsprechend idealtypische Adaptionsoptionen zu erarbeiten, die zukünftige Reallabore nutzen können. Schäpke, Stelzer, Bergmann et al. (2017: 26) betonen, dass bei Transferbemühungen mögliche Nebenfolgen dieser auf andere Untersuchungskontexte zu antizipieren sind. Insbesondere, „wenn das Reallabor und diesbezügliche Experimente in Kontexten mit abweichenden Charakteristika durchgeführt werden sollen (beim sogenannten, scaling out ${ }^{*}$ ), oder wenn durch den Transfer auf neue Systemebenen (beim sogenannten ,scaling up') Experimente und Reallabor mit veränderten Dynamiken konfrontiert werden" (Schäpke/Stelzer/ Bergmann et al. 2017: 27). Hier können Anleihen bei der Technikfolgenabschätzung (Hiller/Krücken 1997; Grunwald 2010; Simonis 2013; Kamlage/Reinermann 2020) für eine noch im Detail zu entwickelnde Evaluationsroutine genommen werden. Die Bindung von Reallaboren an spezifische Kontexte erschwert Transfer und Skalierung (Kügler/
Ronnenberg 2017: 25). Teilnehmende an Reallaboren benennen die Herausforderung, exportierbare, reproduzierbare ,Labor'-Ergebnisse zu generieren und zugleich auf die Bedürfnisse einer nachhaltigen regionalen Entwicklung einzugehen (Engels/Rogge 2018: 30). Grunwald, Schäfer und Bergmann (2020: 109) stellen im Vergleich zu anderen neuen Formaten dar, dass bei Reallaboren Übertragbarkeit ,nur eine untergeordnete Rolle“ spiele. Dies sollte aber nicht bedeuten, dass aus good und bad practice nicht für folgende Projekte gelernt werden dürfte. Weitere Forschung sei erforderlich, um Effizienz und Effektivität gewonnener Erkenntnisse für die Anwendung dieser in unterschiedlichen Kontexten ermitteln zu können (Hintz/Luederitz/Lang et al. 2018: 723).

\subsection{Beitrag zur Transformation}

Die Ergebnisse zum Beitrag zur Transformation legen die Einschätzungen des transformativen Potenzials der einzelnen Reallabore bzw. der dort entwickelten Lösungen und Innovationen sowie die Ziele offen (vgl. Tabelle 4).

Das Anstoßen von Transformationsprozessen zählt zu den Praxiszielen von Reallaboren (Beecroft/Trenks/Rhodius et al. 2018: 78). Das Ziel konnte bei fast allen Reallaboren - nach Aussage der Befragten und Ergebnisdarstellung der Berichte - erfüllt werden $(3$ von $11=$ stimme zu, 7 von 11 = stimme eher zu, 1 von 11 = stimme eher nicht zu). Die Mehrheit der Befragten befand, dass über das Anstoßen von langfristigen Transformationsprozessen keine validen Aussagen getroffen werden können $(1$ von $10=$ stimme zu, 6 
Tabelle 5 Reflexivität und Lernprozesse (Daten aus Fragebögen)

\begin{tabular}{|c|c|c|c|c|}
\hline \multicolumn{5}{|l|}{ Reflexivität und Lernprozesse } \\
\hline & stimme zu & $\begin{array}{l}\text { stimme eher } \\
\text { zu }\end{array}$ & $\begin{array}{l}\text { stimme eher } \\
\text { nicht zu }\end{array}$ & $\begin{array}{l}\text { stimme } \\
\text { nicht zu }\end{array}$ \\
\hline $\begin{array}{l}\text { Es wurden Lernprozesse angestoßen, die in hohem Maße in das Design } \\
\text { integriert wurden. }\end{array}$ & 3 & 7 & 0 & 0 \\
\hline $\begin{array}{l}\text { Die zeitliche Fixierung des Projektrahmens erschwerte es, Anpassun- } \\
\text { gen in den einzelnen Projektphasen vorzunehmen. }\end{array}$ & 5 & 3 & 3 & 1 \\
\hline $\begin{array}{l}\text { Der projektförmige Rahmen des Reallabors wirkte sich nicht hemmend } \\
\text { auf Anpassungsmaßnahmen in den Projektphasen aus. }\end{array}$ & 1 & 1 & 6 & 3 \\
\hline
\end{tabular}

von $10=$ stimme eher $\mathrm{zu}, 3$ von $10=$ stimme eher nicht zu). Inhaltlich deutet dies auf die potenzielle Ungleichzeitigkeit von dem Projektende und dem etwaig angestoßenen transformativen Prozess hin und einer dadurch zwangsläufig schwer zu prognostizierenden Entwicklung und langfristigen Wirkung. Dies spräche für Folgeprojekte, in denen nach einem adäquaten Zeitraum Nacherhebungen durchzuführen wären.

Das in der transformativen Forschung inhärente Ziel einer konkreten Problemlösung (als Beitrag zur Transformation) wurde von sieben Befragten als solches benannt ( $3=$ stimme zu, 4 = stimme eher zu), in vier Fällen kann dies nicht bestätigt werden $(3=$ stimme eher nicht $\mathrm{zu}, 1=$ stimme nicht zu). Der transformative Anteil wurde demnach nicht in allen Fällen an eine Problemlösung gekoppelt. Die Hälfte der Befragten gab an, dass die wissenschaftlichen Ziele nicht gänzlich mit den Praxiszielen ausbalanciert werden konnten $(5$ von $10=$ stimme eher $\mathrm{zu}, 4$ von $10=$ stimme eher nicht zu, 1 von $10=$ stimme nicht zu). Deutlich werden hier Divergenzen, die illustrieren, wie komplex und kompliziert eine Zusammenführung der einzelnen Ziele sein kann. In der Praxis kann beispielsweise der Umstand eintreten, dass Themen im Prozess der Problemerfassung verändert werden. Dies kann dazu führen, dass die gewonnenen Daten keinen Beitrag mehr zur originären Forschungsfrage bzw. zum ,wissenschaftlichen Fortschritt“ (Defila/Di Giulio 2018: 25) leisten können. Unter Praxisziele können noch weitere Elemente gefasst werden (Beecroft/Trenks/Rhodius et al. 2018: 80), wie sie beispielsweise in den Kategorien der praktischen Verstetigung (Anwendung von Lösungen) und der kooperativen Verstetigung (Aktivierung von Personen, Bildung von Kooperationen) aufgeführt werden.

\subsection{Reflexivität und Lernprozesse}

Reallabore sind in ihrer methodologischen Konstitution bereits im Grundsatz reflexiv und experimentell angelegt (Beecroft/Trenks/Rhodius et al. 2018: 83). Lernprozesse werden angestoßen (z. B. im Zuge von Realexperimenten, Interventionen, Erprobungsphasen) und wirken wiederum auf das Forschungsformat und die Forschungsschritte (Ko-De- sign, Ko-Produktion, Ko-Evaluation) in Form von Anpassungen, Reflexionen und Neujustierungen (Rose/Wanner/ Hilger et al. 2019: 7) zurück. Diese dynamischen Wirkmechanismen können in der Projektrealität zu Herausforderungen führen: Wie wirkt sich der Projektrahmen auf derartige (Lern)prozesse, Anpassungen und schlussendlich eine Verstetigung aus (vgl. Tabelle 5)?

Von allen Befragten wurde angegeben, dass im Rahmen der jeweiligen Reallabore des Datenkorpus Lernprozesse angestoßen worden sind, welche in hohem Maße in das Design integriert wurden $(3$ von $10=$ stimme $\mathrm{zu}, 7$ von 10 = stimme eher zu). Die zeitliche Fixierung des Projektrahmens erschwerte Anpassungen in den Projektphasen in acht von 12 Fällen ( 5 von $12=$ stimme $\mathrm{zu}, 3$ von $12=$ stimme eher $\mathrm{zu}, 3$ von 12 = stimme eher nicht $\mathrm{zu}, 1$ von $12=$ stimme nicht $\mathrm{zu}$ ). In nur zwei von elf Fällen wirkte sich die Projektförmigkeit nicht hemmend auf Anpassungsmaßnahmen aus. In den Antworten der offenen Fragen wurde dies expliziert, indem auf die Kürze der Projektzeit (viermal), das begrenzte Budget (dreimal) sowie formal-bürokratische Hürden (einmal) hingewiesen wurde. Die Dynamik der Methode erfordert Flexibilität, lässt keine oder kaum Antragsdisziplin zu und erfordert so eine enge Zusammenarbeit zwischen Geldgebern bzw. Projektträgern und den Projektmitarbeitenden. Dies kann ein Grund für formal-bürokratische Hürden sein. Bezogen auf das Ziel einer Verstetigung der Reallaborlösungen ist davon auszugehen, dass Anpassungen relevant für eine Zusammenarbeit auf Augenhöhe sind. Wenn die Beteiligten erfahren, dass deren Input und Wissen Einfluss auf die Projektphasen und -inhalte nimmt, wird deren Selbstwirksamkeit(sgefühl) gestärkt, ein faires Miteinander möglich und damit die Chance einer langfristigen Verstetigung erhöht (wie auch in der kooperativen Verstetigungskategorie unter Empowerment und Aktivierung aufgeführt) (Eckart/Ley/Häußler et al. 2018: 107108; Räuchle/Schmiz 2019: 14; Best 2020: 96).

\subsection{Transdisziplinarität}

Die Ergebnisse zur Kernkategorie Transdisziplinarität (Schäpke/Stelzer/Bergmann et al. 2017: 19) ermöglichen 
Tabelle 6 Beteiligung der unterschiedlichen Praxisakteure in den unterschiedlichen Projektphasen (Daten aus Fragebögen und Berichten)

\begin{tabular}{lllll}
\hline Welche Akteure waren in welcher Projektphase involviert? & & & \\
\hline & $\begin{array}{l}\text { Formulierung der } \\
\text { Problemstellung }\end{array}$ & $\begin{array}{l}\text { Konzeption des } \\
\text { Reallabors }\end{array}$ & $\begin{array}{l}\text { Durchführung des } \\
\text { Reallabors }\end{array}$ & $\begin{array}{c}\text { Evaluation und } \\
\text { Verbesserung }\end{array}$ \\
\hline Wissenschaftler/-innen & 13 & 12 & 12 & 12 \\
Vertreter/-innen aus Zivilgesellschaft & 7 & 6 & 11 & 7 \\
Vertreter/-innen aus Wirtschaft & 6 & 4 & 7 & 5 \\
Vertreter/-innen aus Politik & 5 & 3 & 6 & 4 \\
Vertreter/-innen aus Verwaltung & 7 & 5 & 9 & 6 \\
Endnutzer/-innen & 2 & 2 & 11 & 7 \\
Sonstige Akteure & 3 & 2 & 4 & 4 \\
\hline
\end{tabular}

Aussagen über die Einbindung von lokalem Wissen, lebensnahen Problemen und außerwissenschaftlichen Praxisakteuren im Forschungsprozess (Schneidewind 2014: 3-4; Defila/Di Giulio 2018: 10). Die Auswertung von Fragebögen und Berichten zeigt die Verteilung der Akteure in den Projektphasen Formulierung der Problemstellung, Konzeption des Reallabors, Durchführung des Reallabors sowie Evaluation und Verbesserung (vgl. Tabelle 6).

Wissenschaftler/-innen waren während allen Phasen beteiligt, wohingegen die Beteiligung anderer Akteure in den unterschiedlichen Phasen variiert. Während der Formulierung der Problemstellung waren vor allem Vertreter/-innen aus Zivilgesellschaft und Verwaltung (jeweils 7 von 13) sowie aus der Wirtschaft (6 von 13) und Politik (5 von 13) involviert. Andere Akteure (3 von 13), darunter aus Verkehrs- und Tarifverbund, Universitäten und Ämtern sowie Endnutzer/-innen ( 2 von 13) waren in wenigen Fällen bereits von Beginn an am Prozess beteiligt. Konzipiert wurden die Reallabore insbesondere in Zusammenarbeit mit Wissenschaftlerinnen/Wissenschaftlern (12 von 13), Vertreterinnen/Vertretern aus Zivilgesellschaft (6 von 13), Verwaltung (5 von 13) und Wirtschaft (4 von 13). Bei der Durchführung des Reallabors waren Vertreter/-innen aus der Zivilgesellschaft und Endnutzer/-innen in elf Fällen, aus Verwaltung in neun, Wirtschaft in sieben und Politik in sechs Fällen beteiligt. Die Phase der Evaluation und Verbesserung fällt ähnlich zur Konzeption der Reallabore aus, mit einem Ausreißer: Die Gruppe der Endnutzer/-innen (7 von 13) wurde hier stärker involviert.

Aus den Projektberichten der Reallabore, zu denen keine Fragebögen vorliegen, kann keine eindeutige Einteilung der Akteurbeteiligung in die einzelnen Phasen erfolgen. Gleichwohl legen die Berichte nahe, dass auch in jenen Projekten Transdisziplinarität einen hohen Stellenwert eingenommen hat und eine enge Kooperation mit Praxisakteuren aus Zivilgesellschaft, Verwaltung, Wirtschaft und Politik erfolgte. Die Auswertung zeigt, dass die Hauptcharakteristika transdisziplinärer Zusammenarbeit (Ko-Design und Ko-Produk- tion von Wissen) (Schäpke/Stelzer/Bergmann et al. 2017: 19) laut einer Mehrheit der Befragten als erfüllt angesehen werden. Die Einbindung der Praxisakteure insbesondere während der Problemformulierung, Durchführung, Evaluation und Verbesserung unterstreicht den transdisziplinären Charakter der Reallabore, welche Wissenschaft und Gesellschaft direkt miteinander zu verknüpfen versuchen (Parodi/ Beecroft/Albiez et al. 2016: 16). Hinsichtlich Verstetigung ermöglicht transdisziplinäre Forschung durch die Einbindung von Praxisakteuren sowie durch den Fokus auf gesellschaftlich relevante Themen ein Ownership der Forschungsprozesse durch nichtwissenschaftliche Akteure (Schäpke/ Stelzer/Bergmann et al. 2017: 24). Durch die Übertragung von Verantwortung für einzelne Maßnahmen und Prozesse kann ein Beitrag geleistet werden, um intrinsische Motivation zu wecken, die eine Verstetigung nach sich ziehen könnte. Eine Herausforderung, welche für das Gelingen transdisziplinärer Zusammenarbeit bewältigt werden muss, ist der Umgang mit unterschiedlichen Wissensformen (Geser 2010), Fähigkeiten, Bedarfen und Bedürfnissen der beteiligten Akteure (Puttrowait/Dietz/Gantert et al. 2018: 201).

\section{Fazit und Ausblick - Reallabore in projektbezogener Umsetzung}

Ob Wissenschaft mithilfe von Reallaboren Veränderungsprozesse in der Lebenswirklichkeit anzustoßen hat, scheint für viele Akteure in der Wissenschaft eine simpel beantwortbare Frage. Entweder sind die Äußerungen begeistert, manchmal wird gar mit Steuermitteln argumentiert, die schließlich in der Lebenswirklichkeit generiert werden und auch dort wieder mit dem Ziel der Mehrwertproduktion eingesetzt werden sollen, oder es wird unter dem Verweis auf eine strikte Aufgabentrennung von Wissenschaft und anderen Systemen der Gesellschaft diese Form experimenteller Forschung abgelehnt. Die Lagerbildung, welche das Aufkommen des Zweigs der transformativen Forschung 
begleitet, darf die Befürworter/-innen jedoch nicht davon abhalten, einen kritischen Blick auf Reallabore zu werfen.

Diese explorative Untersuchung soll einen Anstoß zu einer tiefergehenden Beschäftigung mit diesem Forschungsformat geben. Es sind noch zahlreiche Fragen offen. Diese beginnen bei einer Evaluation, die die Befragung der Praxispartner/-innen einschließt, gehen über eine weitere theoretisch-konzeptionelle Befassung mit Reallaboren hinaus und enden sicher nicht bei der Frage, inwieweit sich diese Methode innerhalb von Organisationen und Institutionen anwenden lässt, um Veränderungsprozesse anzustoßen. In dieser Untersuchung verortet sich unsere Forschungsfrage in den Sphären der Wissenschaft und der Lebenswelt. Beide Sphären prägen die Phasen und Umsetzungsweisen von Reallaboren in einem wechselseitig reflexiven Prozess. Die Gegenüberstellung der konzeptionellen Inhalte und Ansprüche an Reallabore und deren Umsetzung im Drittmittelkontext bildet mögliche Spannungsfelder ab, welche insbesondere beim Fokus auf Aspekte der Verstetigung von primärem Interesse für die Autorinnen und Autoren dieses Beitrags sind. Durch eine kategoriale Annäherung an den Verstetigungsbegriff wurden ausgewählte Drittmittelprojekte hinsichtlich ihrer Verstetigung in der Zusammenschau mit Kernkategorien von Reallaboren untersucht.

Die Ergebnisse weisen einige Auffälligkeiten förderlicher und hemmender Bedingungen auf, die hier nochmals gesondert diskutiert werden. Die untersuchten Ausprägungen zur wissenschaftlichen wie auch praktischen Verstetigung zeigen Spezifika, welche unter anderem in die Forschungslogik transdisziplinärer Projektarbeit eingebettet sind. Wissenschaftler/-innen müssen sich bei der Konzeption, Planung und Durchführung von Reallaboren Herausforderungen stellen, die sich Forschende außerhalb der transformativen Wissenschaft in dem Ausmaß nicht zu stellen haben und für die sie in der Regel auch nicht ausgebildet wurden: Öffentlichkeitsarbeit, Veranstaltungsplanung, Moderation, Motivation und Begeisterung wecken, allgemeinverständliche Wissenschaftsvermittlung und Mediation bei Konflikten sind Aufgaben, die oftmals dazu führen, dass der wissenschaftliche Output - insbesondere in Form von Aufsätzen im PeerReview-Verfahren - in Relation zu anderen Publikationsformen geringer ausfällt. Dies kann sich negativ auf die individuellen wissenschaftlichen Karrieren auswirken. Für eine Verstetigung von Reallaboren bedarf es einer Verstetigung des wissenschaftlichen Arbeitens. Es braucht Lösungen, z. B. fest verankerte Stellen in den Instituten, in denen Forschende gemeinsam mit Bürgerinnen und Bürgern die relevanten Themen der Gegenwart bearbeiten und dies auch in der Lehre verankern und festigen wollen. Beispiele für ambitionierte Verankerungen dieser Forschungslogik in Strukturen von Instituten gab es auch in den untersuchten Projekten. Soll die Arbeit mit Reallaboren professiona- lisiert und Lernkurven methodisch steil gehalten werden, erscheint uns diese Verankerung hilfreich. Ein positives Ergebnis stellt die Generierung von erfolgreichen Folgeanträgen dar.

Darüber hinaus wurden der zeitlichen Fixierung des Projektrahmens hemmende Effekte auf das Forschungsformat Reallabor zugeschrieben, welche Anpassungen und Neujustierungen in den einzelnen Projektphasen erschweren. Für eine transdisziplinäre Zusammenarbeit, welche eine kooperative Verstetigung im Sinne eines Empowerments der Beteiligten befördert, sind die kurzen Projektlaufzeiten kritisch zu reflektieren und im Idealfall von Seiten der Fördergeber grundlegend länger aufzustellen. ${ }^{15}$ Dieses Problem wird im Hinblick auf unseren Datenkorpus meist in Form von Anschlussförderungen gelöst. Kritisch zu bedenken ist an dieser Stelle, inwieweit Anschlussförderungen als strukturelle Verstetigung zu verstehen sind; im eigentlichen Sinne kann hier nur von einer Verlängerung des Reallaborformats im Drittmittelkontext gesprochen werden. Die Beantragungen von Verlängerungen bedeuten Verwaltungsaufwand und kosten Zeit, die der Forschung verloren geht. Die Verstetigung von Reallaboren und deren Lösungen bilden ein zentrales Element transformativer Wissenschaft ab, für welches Evaluationsmethoden (wie bereits beispielhaft durch die Begleitforschung der BaWü-Labs in Angriff genommen) im Rahmen eines heterogenen Anwendungsspektrums zu schärfen und in das Forschungsformat Reallabor einzupflegen sind.

Danksagung Die Untersuchung erfolgte im Rahmen der Projekte „EnerTrend - Systemische Analyse von Wechselwirkungen der Energiewende in NRW mit zentralen Megatrends" (Zuwendung vom Ministerium für Wirtschaft, Innovation, Digitalisierung und Energie des Landes Nordrhein-Westfalen), „PlastikBudget - Entwicklung von Budgetansatz und LCA-Wirkungsabschätzungsmethodik für die Governance von Plastik in der Umwelt" (Zuwendung vom Bundesministerium für Bildung und Forschung) und „JaC-Lab - Integriertes regionales Klimalabor Nord-Jakarta und Hafen“ (Zuwendung vom Bundesministerium für Bildung und Forschung). Wir bedanken uns bei den Fördermittelgebern sowie bei dem Leiter unserer Arbeitsgruppe „Gesellschaft und Nachhaltigkeit im Wandel“ (Geographisches Institut, Ruhr-Universität Bochum), Prof. Dr. Andreas Farwick.

\section{Literatur}

Arnstein, S. R. (1969): A ladder of citizen participation. In: Journal of the American Institute of Planners 35, 4, 216-

224. https://doi.org/10.1080/01944366908977225

Augé, M. (2011): Nicht-Orte. Nördlingen.

\footnotetext{
15 Eine spezifische Darstellung wissenschaftspolitischer Empfehlungen, in der ähnliche (Heraus-)Forderungen formuliert werden, findet sich bei Defila und Di Giulio (2019).
} 
Beecroft, R.; Parodi, O. (2016): Reallabore als Orte der Nachhaltigkeitsforschung und Transformation. In: Zeitschrift für Technikfolgenabschätzung in Theorie und Praxis 25, 3, 4-8. https://doi.org/10.14512/tatup.25.3.4

Beecroft, R.; Trenks, H.; Rhodius, R.; Benighaus, C.; Parodi, O. (2018): Reallabore als Rahmen transformativer und transdisziplinärer Forschung: Ziele und Designprinzipien. In: Defila, R.; Di Giulio, A. (Hrsg.): Transdisziplinär und transformativ forschen. Eine Methodensammlung. Wiesbaden, 75-100. https://doi.org/10.1007/9783-658-21530-9_4

Best, B. (2020): Experimentieren erlaubt: Reallabore in Forschung und Praxis. In: Politische Ökologie 38, 160, 9399.

Brinkmann, C.; Bergmann, M.; Huang-Lachmann, J.-T.; Rödder, S.; Schuck-Zöller, S. (2015): Zur Integration von Wissenschaft und Praxis als Forschungsmodus. Ein Literaturüberblick. Hamburg. = Climate Service Center Report 23.

Defila, R.; Di Giulio, A. (2018): Reallabore als Quelle für die Methodik transdisziplinären und transformativen Forschens - eine Einführung. In: Defila, R.; Di Giulio, A. (Hrsg.): Transdisziplinär und transformativ forschen. Eine Methodensammlung. Wiesbaden, 9-35. https://doi. org/10.1007/978-3-658-21530-9_1

Defila, R.; Di Giulio, A. (2019): Wissenschaftspolitische Empfehlungen zum Forschungs- und Förderformat Reallabor. Basel.

Deutscher Bundestag (2018): Förderung von Reallaboren und Citizen Science in Deutschland. Dokumentation. Wissenschaftliche Dienste des Deutschen Bundestages. https://www.bundestag.de/resource/blob/550742/ 8f269b6399b3098eabf9d5b09f31f88f/WD-8-017-18pdf-data.pdf (12.03.2021).

Diethard, M.; Höflehner, T. (2018): Urban Labs als Trend für eine nachhaltige Stadtentwicklung. In: Geograz 63, 11-14.

Döring, N.; Bortz, J. (2016): Forschungsmethoden und Evaluation in den Sozial- und Humanwissenschaften. Berlin. https://doi.org/10.1007/978-3-642-41089-5

Eckart, J.; Ley, A.; Häußler, E.; Erl, T. (2018): Leitfragen für die Gestaltung von Partizipationsprozessen in Reallaboren. In: Defila, R.; Di Giulio, A. (Hrsg.): Transdisziplinär und transformativ forschen. Eine Methodensammlung. Wiesbaden, 105-135. https://doi.org/10.1007/9783-658-21530-9_6

Engels, F.; Rogge, J.-C. (2018): Tensions and Trade-offs in Real-World Laboratories - The Participants' Perspective. In: GAIA - Ecological Perspectives for Science and Society 27, S1, 28-31. https://doi.org/10.14512/gaia.27.S1. 8

Freier, A. N.; Lübke, S.; Schrot, K.; Zorn, J. (2020):
Empowerment in Transformations-Arenen. Berücksichtigung sozialer Disparitäten im Energiewendeprozess. In: Engler, S.; Janik, J.; Wolf, M. (Hrsg.): Energiewende und Megatrends. Wechselwirkungen von globaler Gesellschaftsentwicklung und Nachhaltigkeit. Bielefeld, 235273. https://doi.org/10.14361/9783839450710

Geels, F. W. (2011): The multi-level perspective on sustainability transitions: Responses to seven criticisms. In: Environmental Innovation and Societal Transitions 1, 1, 24-40. https://doi.org/10.1016/j.eist.2011.02.002

Geels, F. W.; Schot, J. (2007): Typology of sociotechnical transition pathways. In: Research Policy 36, 3, 399-417. https://doi.org/10.1016/j.respol.2007.01.003

Geser, H. (2010): Wissensformen, Arbeitsrollen und Kooperationsstrukturen. Ein funktional-morphologischer Theorieansatz. Zürich. https://www.geser.net/arbeit/t_ hgeser7.pdf (12.03.2021).

Grin, J.; Rotmans, J.; Schot, J. (2010): Transitions to Sustainable Development. New Directions in the Study of Long Term Transformative Change. New York.

Grunwald, A. (2010): Technikfolgenabschätzung: Eine Einführung. Berlin.

Grunwald, A. (2015): Transformative Wissenschaft - eine neue Ordnung im Wissenschaftsbetrieb? In: GAIA - Ecological Perspectives for Science and Society 24, 1, 1720. https://doi.org/10.14512/gaia.24.1.5

Grunwald, A.; Schäfer, M.; Bergmann, M. (2020): Neue Formate transdisziplinärer Forschung: Ausdifferenzierte Brücken zwischen Wissenschaft und Praxis. In: GAIA Ecological Perspectives for Science and Society 29, 2, 106-114. https://doi.org/10.14512/gaia.29.2.8

Harvey, D. (2013): Verdichtung von Raum. In: Günzel, S. (Hrsg.): Texte zur Theorie des Raums. Stuttgart, 89-93.

Hiller, P.; Krücken, G. (Hrsg.) (1997): Risiko und Regulierung. Soziologische Beiträge zu Technikkontrolle und präventiver Umweltpolitik. Frankfurt am Main.

Hintz, M. J.; Luederitz, C.; Lang, D. J.; von Wehrden, H. (2018): Facing the heat: A systematic literature review exploring the transferability of solutions to cope with urban heat waves. In: Urban Climate 24, 714-727. https:// doi.org/10.1016/j.uclim.2017.08.011

Jahn, T. (2013): Transdisziplinarität - Forschungsmodus für nachhaltiges Forschen. In: Hacker, J. (Hrsg.): Nachhaltigkeit in der Wissenschaft. Stuttgart, 65-75. = Nova Acta Leopoldina 398.

Kamlage, J.-H.; Reinermann, J. L. (2020): Die doppelte Normativität der partizipativen Technikfolgenabschätzung - Entwicklungen, Funktionen und Herausforderungen. In: Nierling, L.; Torgersen, H. (Hrsg.): Die neutrale Normativität der Technikfolgenabschätzung. Konzeptionelle Auseinandersetzung und praktischer 
Umgang. Baden-Baden, 39-56. https://doi.org/10.5771/ 9783748907275-39

Karvounis, A. (2015): Urban metabolism. In: Chrysoulakis, N.; de Castro, A. E.; Moors, E. J. (Hrsg.): Understanding Urban Metabolism. A tool for urban planning. London, 3-12.

Kügler, V.; Ronnenberg, P. (2017): Reallabore. Kurzzeitiger Trend oder innovatives Instrument zur resilienten Stadtentwicklung? In: Raum \& Resilienz: Zukunft von Stadtregionen 2, 22-27.

Mayring, P. (2010): Qualitative Inhaltsanalyse. Weinheim.

Meyer-Soylu, S.; Parodi, O.; Trenks, H.; Seebacher, A. (2016): Das Reallabor als Partizipationskontinuum. Erfahrungen aus dem Quartier Zukunft und Reallabor 131 in Karlsruhe. In: Technikfolgenabschätzung in Theorie und Praxis 25, 3, 31-40. https://doi.org/10.14512/tatup. 25.3.31

Parodi, O.; Beecroft, R.; Albiez, M.; Quint, A.; Seebacher, A.; Tamm, K.; Waitz, C. (2016): Von „Aktionsforschung“ bis „Zielkonflikte“. Schlüsselbegriffe der Reallaborforschung. In: Technikfolgenabschätzung in Theorie und Praxis 25, 3, 9-18. https://doi.org/10.14512/tatup. 25.3.9

Puttrowait, E.; Dietz, R.; Gantert, M.; Heynold, J. (2018): Der Weg zum Realexperiment - Schlüsselakteure identifizieren, Kooperationsstrukturen aufbauen, Projektideen auswählen. In: Defila, R.; Di Giulio, A. (Hrsg.): Transdisziplinär und transformativ forschen. Eine Methodensammlung. Wiesbaden, 195-232. https://doi.org/ 10.1007/978-3-658-21530-9_11

Räuchle, C.; Schmiz, A. (2019): Reallabore in Ankunftsquartieren. Osnabrück. = IMIS Working Paper 03, Institut für Migrationsforschung und Interkulturelle Studien der Universität Osnabrück.

Rose, M.; Wanner, M.; Hilger, A. (2019): Das Reallabor als Forschungsprozess und -infrastruktur für nachhaltige Entwicklung. Konzepte, Herausforderungen und Empfehlungen. o.O. = NaWiKo Synthese Working Paper 1.

Schäpke, N.; Stelzer, F.; Bergmann, M.; Singer-Brodowski, M.; Wanner, M.; Caniglia, G.; Lang, D. J. (2017): Reallabore im Kontext transformativer Forschung. Ansatzpunkte zur Konzeption und Einbettung in den internationalen Forschungsstand. Lüneburg. = IETSR Discussion Papers in Transdisciplinary Sustainability Research 1/2017.

Schäpke, N.; Stelzer, F.; Caniglia, G.; Bergmann, M.; Wanner, M.; Singer-Brodowski, M.; Loorbach, D.; Olsson, P.; Baedeker, C.; Lang, D. J. (2018): Jointly Experimenting for Transformation? Shaping Real-World Laboratories by Comparing Them. GAIA - Ecological Perspectives for
Science and Society 27, S1, 85-96. https://doi.org/10. 14512/gaia.27.S1.16

Schneidewind, U. (2014): Urbane Reallabore - ein Blick in die aktuelle Forschungswerkstatt. In: pnd|online 3, 1-7.

Schneidewind, U. (2015): Transformative Wissenschaft Motor für gute Wissenschaft und lebendige Demokratie. In: GAIA - Ecological Perspectives for Science and Society 24, 2, 88-91. https://doi.org/10.14512/gaia.24.2.5

Schneidewind, U.; Singer-Brodowski, M. (2014): Transformative Wissenschaft. Klimawandel im deutschen Wissenschafts- und Hochschulsystem. Marburg.

Schneidewind, U.; Singer-Brodowski, M. (2015): Vom experimentellen Lernen zum transformativen Experimentieren - Reallabore als Katalysator für eine lernende Gesellschaft auf dem Weg zu einer Nachhaltigen Entwicklung. In: Zeitschrift für Wirtschafts- und Unternehmensethik 16, 1, 10-23. https://doi.org/10.5771/1439-880X2015-1-10

Schreier, M.; Odağ, Ö. (2010): Mixed Methods. In: Mey, G.; Mruck, K. (Hrsg.): Handbuch Qualitative Forschung in der Psychologie. Wiesbaden, 263-277. https://doi.org/ 10.1007/978-3-531-92052-8_18

Simonis, G. (Hrsg.) (2013): Konzepte und Verfahren der Technikfolgenabschätzung. Wiesbaden. https://doi.org/ 10.1007/978-3-658-02035-4

Strohschneider, P. (2014): Zur Politik der Transformativen Wissenschaft. In: Brodocz, A.; Herrmann, D.; Schmidt, R.; Schulz, D.; Schulze Wessel, J. (Hrsg.): Die Verfassung des Politischen. Festschrift für Hans Vorländer. Wiesbaden, 175-192. https://doi.org/10.1007/9783-658-04784-9_10

Wagner, F. (2017): Reallabore als kreative Arenen der Transformation zu einer Kultur der Nachhaltigkeit. In: Reinermann, J.-L.; Behr, F. (Hrsg.): Die Experimentalstadt. Kreativität und die kulturelle Dimension der Nachhaltigen Entwicklung. Wiesbaden, 79-94. https://doi.org/10. 1007/978-3-658-14981-9_5

Wanner, M.; Stelzer, F. (2019): Reallabore - Perspektiven für ein Forschungsformat im Aufwind. Wuppertal. = In Brief 07/2019.

WBGU - Wissenschaftlicher Beirat der Bundesregierung Globale Umweltveränderungen (2011): Welt im Wandel. Gesellschaftsvertrag für eine Große Transformation. Berlin.

WBGU - Wissenschaftlicher Beirat der Bundesregierung Globale Umweltveränderungen (2016): Der Umzug der Menschheit. Die transformative Kraft der Städte. Berlin. Wissenschaftsrat (2015): Zum wissenschaftspolitischen Diskurs über Große gesellschaftliche Herausforderungen. Positionspapier. Stuttgart. 